Eng. Appl. Sci. Lett., Vol. 1(2018), No. 1, pp. 01 - 09

Website: https://pisrt.org/psr-press/journals/easl/

ISSN: 2617-9709 (Online) 2617-9695 (Print)

http://dx.doi.org/10.30538/psrp-easl2018.0001

\title{
SOME NUMERICAL INVARIANTS ASSOCIATED WITH V-PHENYLENIC NANOTUBE AND NANOTORI
}

\author{
RACHANNA KANABUR ${ }^{1}$, SUNILKUMAR HOSAMANI
}

\begin{abstract}
A carbon nanotube (CNT) is a miniature cylindrical carbon structure that has hexagonal graphite molecules attached at the edges. In this paper, we compute the numerical invariant (Topological indices) of linear [n]-phenylenic, lattice of $C_{4} C_{6} C_{8}[m, n], T U C_{4} C_{6} C_{8}[m, n]$ nanotube, $C_{4} C_{6} C_{8}[m, n]$ nanotori.
\end{abstract}

Index Terms: Molecular graph; topological index; nanotube; nanotori.

\section{Introduction}

Mathematical chemistry is a branch of theoretical chemistry for discussion and prediction of the molecular structure using mathematical methods without necessarily referring to quantum mechanics. Chemical graph theory is a branch of mathematical chemistry which applies graph theory to mathematical modelling of chemical phenomena [1, 2]. This theory had an important effect on the development of the chemical sciences. In mathematics chemistry, a molecular graph is a simple graph such that its vertices correspond to the atoms and the edges to the bonds. And also a connected graph is a graph such that there is a path between all pairs of vertices. Note that hydrogen atoms are often omitted [2]. Let $G=(V, E)$ be a graph with n vertices and $m$ edges. The degree of a vertex $u \in V(G)$ is denoted by $d_{G}(u)$ and is the number of vertices that are adjacent to $u$. The edge connecting the vertices $u$ and $v$ is denoted by $u v[3]$.

\footnotetext{
Received 28-08-2018. Revised 01-09-2018. Accepted 20-09-2018.

1 Corresponding Author

(C) 2018 Rachanna Kanabur, Sunilkumar Hosamani. This is an open access article distributed under the Creative Commons Attribution License, which permits unrestricted use, distribution, and reproduction in any medium, provided the original work is properly cited.
} 


\section{Computing the Topological Indices of Certain Nanotubes}

In 4, 5, 6, Shigehalli and Kanabur have put forward new degree based topological indices viz. arithmetic-geometric index, $S K$ index, $S K_{1}$ index and $S K_{2}$ index. Which are defined as follows: Let $G=(V, E)$ be a molecular graph, $d_{G}(u)$ and $d_{G}(v)$ is the degree of the vertex $u$ and $v$, then

$$
\begin{gathered}
A G_{1}=\sum_{u v \in E(G)} \frac{1}{2 \sqrt{d_{u}+d_{v}}}, \\
S K=\sum_{u v \in E(G)} \frac{d_{u}+d_{v}}{2}, \\
S K_{1}=\sum_{u v \in E(G)} \frac{d_{u} d_{v}}{2}, \\
S K_{2}=\sum_{u v \in E(G)}\left(\frac{d_{u}+d_{v}}{2}\right)^{2} .
\end{gathered}
$$

where $d_{G}(u)$ and $d_{G}(v)$ are the degrees of the vertices $u$ and $v$ in $G$. In this paper we give explicit formulae for these topological indices of [n]-phenylenic, lattice of $C_{4} C_{6} C_{8}[m, n], T U C_{4} C_{6} C_{8}[m, n]$ nanotube, $C_{4} C_{6} C_{8}[m, n]$ nanotori [7] 8 .

\section{Main Results}

The aim of this section, at first, is to compute some topological indices of the molecular graph of linear[n]-phenylenic as depicted in Fig.1



Figure 1. The molecular graph of a linear [n]-phenylenic.

It is easy to see that $T=T[n]$ has $6 n$ vertices and $8 n-2$ edges, We partition the edges of $T$ into three subsets $E_{1}(T), E_{2}(T)$ and $E_{3}(T)$, Table1 shows the number of three types of edges.

TABLE 1. The number of three types of edges of the graph $T$.

\begin{tabular}{|l|l|}
\hline$\left(d_{u}, d_{v}\right)$ & Number of edges \\
\hline$(2,2)$ & 6 \\
\hline$(2,3)$ & $4 n-4$ \\
\hline$(3,3)$ & $4 n-4$ \\
\hline
\end{tabular}

From this table, we given an explicit computing formula for some indices of a linear [n]-phenylenic, as shown in above graph.

Theorem 3.1. Consider the graph $T$ of a linear $[n]-p h e n y l e n i c$. Then the $A G_{1}$, $S K, S K_{1}$ and $S K_{2}$ indices of $T$ are equal to 
(1) $A G_{1}(G)=8.08 n-2.08$,

(2) $S K(G)=22 n-10$,

(3) $S K_{1}(G)=30 n-18$,

(4) $S K_{2}(G)=61 n-37$.

Proof.

(1)

$$
\begin{aligned}
A G_{1}(G)= & \sum_{u v \in E(G)} \frac{d_{u}+d_{v}}{2 \sqrt{d_{u} \cdot d_{v}}} \\
= & \sum_{u v \in E_{1}(G)} \frac{d_{u}+d_{v}}{2 \sqrt{d_{u} \cdot d_{v}}}+\sum_{u v \in E_{2}(G)} \frac{d_{u}+d_{v}}{2 \sqrt{d_{u} \cdot d_{v}}} \\
& +\sum_{u v \in E_{3}(G)} \frac{d_{u}+d_{v}}{2 \sqrt{d_{u} \cdot d_{v}}} \\
= & \left|E_{1}(G)\right| \frac{2+2}{2 \sqrt{2.2}}+\left|E_{2}(G)\right| \frac{2+3}{2 \sqrt{2.3}} \\
& +\left|E_{3}(G)\right| \frac{3+3}{2 \sqrt{3.3}} \\
= & 6(1)+(4 n-4)\left(\frac{5}{2 \sqrt{6}}\right)+(4 n-4)(1) \\
= & 8.08 n-2.08 .
\end{aligned}
$$

(2)

$$
\begin{aligned}
S K(G)= & \sum_{u v \in E(G)} \frac{d_{u}+d_{v}}{2} \\
= & \sum_{u v \in E_{1}(G)} \frac{d_{u}+d_{v}}{2}+\sum_{u v \in E_{2}(G)} \frac{d_{u}+d_{v}}{2} \\
& +\sum_{u v \in E_{3}(G)} \frac{d_{u}+d_{v}}{2} \\
= & \left|E_{1}(G)\right| \frac{2+2}{2}+\left|E_{2}(G)\right| \frac{2+3}{2} \\
& +\left|E_{3}(G)\right| \frac{3+3}{2} \\
= & 12+10 n-10+12 n-12 \\
= & 22 n-10 .
\end{aligned}
$$

(3)

$$
S K_{1}(G)=\sum_{u v \in E(G)} \frac{d_{u} d_{v}}{2}
$$




$$
\begin{aligned}
= & \sum_{u v \in E_{1}(G)} \frac{d_{u} d_{v}}{2}+\sum_{u v \in E_{2}(G)} \frac{d_{u} d_{v}}{2} \\
& +\sum_{u v \in E_{3}(G)} \frac{d_{u} d_{v}}{2} \\
= & \left|E_{1}(G)\right| \frac{2.2}{2}+\left|E_{2}(G)\right| \frac{2.3}{2} \\
& +\left|E_{3}(G)\right| \frac{3.3}{2} \\
= & =12+12 n-12+18 n-18 \\
= & 30 n-18 .
\end{aligned}
$$

(4)

$$
\begin{aligned}
S K_{2}(G)= & \sum_{u v \in E(G)}\left(\frac{d_{u}+d_{v}}{2}\right)^{2} \\
= & \sum_{u v \in E_{1}(G)}\left(\frac{d_{u}+d_{v}}{2}\right)^{2}+\sum_{u v \in E_{2}(G)}\left(\frac{d_{u}+d_{v}}{2}\right)^{2} \\
& +\sum_{u v \in E_{3}(G)}\left(\frac{d_{u}+d_{v}}{2}\right)^{2} \\
= & \left|E_{1}(G)\right|\left(\frac{2+2}{2}\right)^{2}+\left|E_{2}(G)\right|\left(\frac{2+3}{2}\right)^{2} \\
& +\left|E_{3}(G)\right|\left(\frac{3+3}{2}\right)^{2} \\
= & 24+25 n-25+36 n-36 \\
= & 61 n-37 .
\end{aligned}
$$

In continue of this section, we see the following figures

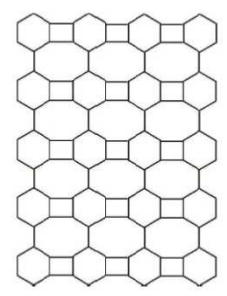

Figure 2. The 2-D graph lattice of $C_{4} C_{6} C_{8}[4,5]$ nanotube.

We now consider the molecular graph $G=C_{4} C_{6} C_{8}[m, n]$, Fig.2. It is easy to see that $|V(G)|=6 m n$ and $|E(G)|=9 m n-2 n-m$, We partition the edges of 
$G$ into three subsets $E_{1}(G), E_{2}(G)$ and $E_{3}(G)$. The number of three types of edges is shown in Table 2 .

TABLE 2. The number of three types of edges of the graph $T$.

\begin{tabular}{|l|l|}
\hline$\left(d_{u}, d_{v}\right)$ & Number of edges \\
\hline$(2,2)$ & $2 n+4$ \\
\hline$(2,3)$ & $4 m+4 n-8$ \\
\hline$(3,3)$ & $9 m n-8 n-5 m+4$ \\
\hline
\end{tabular}

From this table, we have given an explicit computing of some indices of $G$ (Fig. 2).

Theorem 3.2. Consider the graph $T$ of a linear $[n]-p h e n y l e n i c$. Then the $A G_{1}$, $S K, S K_{1}$ and $S K_{2}$ indices of $T$ are equal to

(1) $A G_{1}(G)=(9 n-5.92) m-9.92 n-3.84$,

(2) $S K(G)=(27 n-5) m-10 n$,

(3) $S K_{1}(G)=(40.5 n-10) m-20 n+2$,

(4) $S K_{2}(G)=(81 n-20) m-39 n-48$.

Proof. (1)

$$
\begin{aligned}
A G_{1}(G)= & \sum_{u v \in E(G)} \frac{d_{u}+d_{v}}{2 \sqrt{d_{u} \cdot d_{v}}} \\
= & \sum_{u v \in E_{1}(G)} \frac{d_{u}+d_{v}}{2 \sqrt{d_{u} \cdot d_{v}}}+\sum_{u v \in E_{2}(G)} \frac{d_{u}+d_{v}}{2 \sqrt{d_{u} \cdot d_{v}}} \\
& +\sum_{u v \in E_{3}(G)} \frac{d_{u}+d_{v}}{2 \sqrt{d_{u} \cdot d_{v}}} \\
= & \left|E_{1}(G)\right| \frac{2+2}{2 \sqrt{2.2}}+\left|E_{2}(G)\right| \frac{2+3}{2 \sqrt{2.3}} \\
& +\left|E_{3}(G)\right| \frac{3+3}{2 \sqrt{3.3}} \\
= & 9 m n-5.92 m-9.92 n-3.04 \\
= & =(9 n-5.92) m-9.92 n-3.84 .
\end{aligned}
$$

(2)

$$
\begin{aligned}
S K(G)= & \sum_{u v \in E(G)} \frac{d_{u}+d_{v}}{2} \\
= & \sum_{u v \in E_{1}(G)} \frac{d_{u}+d_{v}}{2}+\sum_{u v \in E_{2}(G)} \frac{d_{u}+d_{v}}{2} \\
& +\sum_{u v \in E_{3}(G)} \frac{d_{u}+d_{v}}{2}
\end{aligned}
$$




$$
\begin{aligned}
= & \left|E_{1}(G)\right| \frac{2+2}{2}+\left|E_{2}(G)\right| \frac{2+3}{2} \\
& +\left|E_{3}(G)\right| \frac{3+3}{2} \\
= & =4 n+8+10 m+10 n-20+27 m n-24 n-15 m+12 \\
= & 27 m n-10 n-5 m .
\end{aligned}
$$

(3)

$$
\begin{aligned}
S K_{1}(G)= & \sum_{u v \in E(G)} \frac{d_{u} d_{v}}{2} \\
= & \sum_{u v \in E_{1}(G)} \frac{d_{u} d_{v}}{2}+\sum_{u v \in E_{2}(G)} \frac{d_{u} d_{v}}{2} \\
& +\sum_{u v \in E_{3}(G)} \frac{d_{u} d_{v}}{2} \\
= & \left|E_{1}(G)\right| \frac{2.2}{2}+\left|E_{2}(G)\right| \frac{2.3}{2} \\
& +\left|E_{3}(G)\right| \frac{3.3}{2} \\
= & =4 n+8+12 m+12 n-24+40.5 m n-36 n-22.5 m+8 \\
= & (40.5 n-10) m-20 n+2 .
\end{aligned}
$$

(4)

$$
\begin{aligned}
S K_{2}(G)= & \sum_{u v \in E(G)}\left(\frac{d_{u}+d_{v}}{2}\right)^{2} \\
= & \sum_{u v \in E_{1}(G)}\left(\frac{d_{u}+d_{v}}{2}\right)^{2}+\sum_{u v \in E_{2}(G)}\left(\frac{d_{u}+d_{v}}{2}\right)^{2} \\
& +\sum_{u v \in E_{3}(G)}\left(\frac{d_{u}+d_{v}}{2}\right)^{2} \\
= & \left|E_{1}(G)\right|\left(\frac{2+2}{2}\right)^{2}+\left|E_{2}(G)\right|\left(\frac{2+3}{2}\right)^{2} \\
& +\left|E_{3}(G)\right|\left(\frac{3+3}{2}\right)^{2} \\
= & 8 n+16+25 m+25 n-100+81 m n-72 n-45 m+36 \\
= & (81 n-20) m-39 n-48 .
\end{aligned}
$$




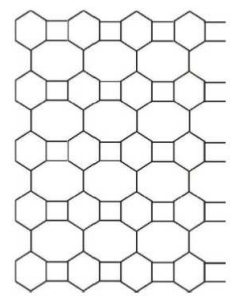

Figure 3. The 2-D graph lattice of $T U C_{4} C_{6} C_{8}[4,5]$ nanotube.

We now consider the molecular graph $K=T U C_{4} C_{6} C_{8}[m, n]$, Fig. 3 . It is easy to see that $|V(K)|=6 m n$ and $|E(K)|=9 m n-n$. We partition the edges of nanotube $K$ into two subsets $E_{1}(G), E_{2}(G)$ and compute the total number of edges for the 2-dimensional of graph $K$ (Table 3 ).

TABLE 3. The number of three types of edges of the graph $T$.

\begin{tabular}{|l|l|}
\hline$\left(d_{u}, d_{v}\right)$ & Number of edges \\
\hline$(2,3)$ & $4 n$ \\
\hline$(3,3)$ & $9 m n-5 m$ \\
\hline
\end{tabular}

From this table, we given an explicit computing formula for some indices of a linear $[\mathrm{n}]$-phenylenic, as shown in above graph.

Theorem 3.3. Consider the graph $T$ of a linear[n]-phenylenic. Then the $A G_{1}$, $S K, S K_{1}$ and $S K_{2}$ indices of $T$ are equal to

(1) $A G_{1}(G)=(9 n-0.92) m$,

(2) $S K(G)=(27 n-5) m$.,

(3) $S K_{1}(G)=(40.5 n-10.5) m$.,

(4) $S K_{2}(G)=(81 n-20) m$..

Proof. (1)

$$
\begin{aligned}
A G_{1}(G) & =\sum_{u v \in E(G)} \frac{d_{u}+d_{v}}{2 \sqrt{d_{u} \cdot d_{v}}} \\
& =\sum_{u v \in E_{1}(G)} \frac{d_{u}+d_{v}}{2 \sqrt{d_{u} \cdot d_{v}}}+\sum_{u v \in E_{2}(G)} \frac{d_{u}+d_{v}}{2 \sqrt{d_{u} \cdot d_{v}}} \\
& =\left|E_{1}(G)\right| \frac{2+3}{2 \sqrt{2.2}}+\left|E_{2}(G)\right| \frac{3+3}{2 \sqrt{2.3}} \\
& =9 m n-5 m+4.08 m \\
& =(9 n-0.92) m .
\end{aligned}
$$

$$
S K(G)=\sum_{u v \in E(G)} \frac{d_{u}+d_{v}}{2}
$$




$$
\begin{aligned}
& =\sum_{u v \in E_{1}(G)} \frac{d_{u}+d_{v}}{2}+\sum_{u v \in E_{2}(G)} \frac{d_{u}+d_{v}}{2} \\
& =\left|E_{1}(G)\right| \frac{2+3}{2}+\left|E_{2}(G)\right| \frac{3+3}{2} \\
& =10 m+27 m n-15 m \\
& =(27 n-5) m .
\end{aligned}
$$

(3)

$$
\begin{aligned}
S K_{1}(G) & =\sum_{u v \in E(G)} \frac{d_{u} d_{v}}{2} \\
& =\sum_{u v \in E_{1}(G)} \frac{d_{u} d_{v}}{2}+\sum_{u v \in E_{2}(G)} \frac{d_{u} d_{v}}{2} \\
& =\left|E_{1}(G)\right| \frac{2.3}{2}+\left|E_{2}(G)\right| \frac{3.3}{2} \\
& ==12 m n+(9 m n-5 m)(4.5) \\
& =(40.5 n-10.5) m .
\end{aligned}
$$

(4)

$$
\begin{aligned}
S K_{2}(G) & =\sum_{u v \in E(G)}\left(\frac{d_{u}+d_{v}}{2}\right)^{2} \\
& =\sum_{u v \in E_{1}(G)}\left(\frac{d_{u}+d_{v}}{2}\right)^{2}+\sum_{u v \in E_{2}(G)}\left(\frac{d_{u}+d_{v}}{2}\right)^{2} \\
& =\left|E_{1}(G)\right|\left(\frac{2+3}{2}\right)^{2}+\left|E_{2}(G)\right|\left(\frac{3+3}{2}\right)^{2} \\
& =25 m+81 m n-45 m \\
& =(81 n-20) m .
\end{aligned}
$$

\section{4. conclusion}

In this paper, we have computed the value of $A G_{1}$ index, $S K$ index, $S K_{1}$ index and $S K_{2}$ index for Linear [n]-phenylenic, lattice of $C_{4} C_{6} C_{8}[m, n], T U C_{4} C_{6} C_{8}[m, n]$ nanotube, $C_{4} C_{6} C_{8}[m, n]$ nanotori without using computer.

\section{Competing Interests}

The authors declare that they have no competing interests. 


\section{REFERENCES}

1. Diudea, M. V., Gutman, I., \& Jantschi, L. (2001). Molecular topology. Huntington, NY: Nova Science Publishers.

2. Trinajstić, N. (1992). Chemical Graph Theory, CRC Press. Boca Raton.

3. Harary, F. (1969). Graph theory. Addison-Wesely, Reading mass.

4. Shigehalli, V. S., \& Kanabur, R. (2016). New Version of Degree-Based Topological Indices of Certain nanotube. J. Math. Nanosci, 6(1), 29-42.

5. Shigehalli, V. S., \& Kanabur, R. (2016). Computation of new degree-based topological indices of graphene. Journal of Mathematics, 2016.

6. Shigehalli, V., \& Kanabur, R. (2016). Computing degree-based topological indices of Polyhex nanotubes. Journal of Mathematical Nanoscience, 6(1-2), 47-55.

7. Nikmehr, M. J., Veylaki, M., \& Soleimani, N. (2015). Some topological indices of VPhenylenic nanotube and nanotori. Optoelectron. Adv. Mater.-Rapid Comm, 9(9), 11471149 .

8. Hosamani, S. M., \& Gutman, I. (2014). Zagreb indices of transformation graphs and total transformation graphs. Applied Mathematics and Computation, 247, 1156-1160.

\section{Rachanna Kanabur}

Department of Mathematics, BLDEA'S Commerce BHS Arts and TGP Science, College, Jamakhandi - 587301 Karnataka, India.

e-mail: rachukanabur@gmail.com

\section{Sunilkumar Hosamani}

Department of Mathematics, Rani Channamma University Belagavi - 591156 Karnataka, India.

e-mail: sunilkumar.rcu@gmail.com 